\title{
CARACTERÍSTICAS PRINCIPALES, LOGROS Y DIFICULTADES DE LA GESTIÓN DE LA INVESTIGACIÓN EN EL NIVEL SUPERIOR Y SU CORRELATO EN LAS PUBLICACIONES
}

\author{
María Isabel Pozzo \\ Docente del Departamento de Investigaciones y Publicaciones en el \\ Instituto de Educación Superior N 28 "Olga Cossettini" \\ Rosario, Argentina
}

Enviado 23 de febrero 2007 • Aprobado 19 de junio 2007

\begin{abstract}
Resumen: En este artículo se presentan las principales características, logros y dificultades de la gestión de la investigación en el nivel superior y su correlato en las publicaciones, a partir de la experiencia transitada en las instituciones de pertenencia de la autora (superior no universitario y urniversitario de grado y posgrado) en Argentina. La presentación persigue como objetivo ulterior el acercamiento interinstitucional a partir del intercambio de estrategias tendientes a favorecer la investigación como factor fundamental de la formación docente.
\end{abstract}

Palabras clave: Investigación, publicaciones, nivel superior, gestión.

\begin{abstract}
In this article, the main characteristics, achievements and difficulties in research and publishing management in higher education are presented, taking into account the experience of the author in the institutions to which she belongs (Teacher Trainning College and Graduate and Post graduate university levels) in Argentina. The presentation seeks to foster institutional exchange of experiences from sharing strategies that may help develop research activities as a key factor in teacher trainning.
\end{abstract}

Key words: Research, publications, higher education, management.

\section{Presentación}

La temática de este artículo persigue hallar puntos en común entre profesores, estudiantes y responsables de Departamentos de Investigación de nivel superior de diversas instituciones y regiones. Dicha amplitud se debe a la posibilidad de encontrar dificultades comunes, sistematizarlas y sugerir propuestas aplicables tanto entre quienes gestionan las investigaciones en el ámbito institucional, como en la clase. En todo caso, a lo largo de la exposición, se señalan las particularidades de

\footnotetext{
Docente del Departamento de Investigaciones y Publicaciones en el Instituto de Educación Superior No 28 "Olga Cossettini” de Rosario, Argentina y Escuela de Ciencias de la Educación de la Universidad Nacional de Rosario, Argentina. Doctorado en Humanidades y Artes.
} 
los distintos planos. A su vez, se intenta que las cuestiones tratadas sean de interés para quienes, sin estar a cargo de un Departamento de Investigaciones ni una cátedra relacionada, puedan tomarlas en el proceso de construcción de una investigación.

\section{NIVELES EN LA GESTIÓN DE LA INVESTIGACIÓN}

En función del espectro de destinatarios de esta temática, aludido en el parágrafo de presentación, podemos hablar de tres niveles en la gestión de la investigación:

- $\quad$ El institucional, encarnado en la mayoría de los casos en los Departamentos de investigaciones y cuyos destinatarios son fundamentalmente los docentes.

- $\quad$ El nivel áulico, conformado por las asignaturas relacionadas con la investigación, llámense "Metodología de la Investigación”, "Seminario de Integración”, Taller de Práctica, etcétera.

- El plano individual: en tanto la mayoría de los docentes se encuentran abocados a la confección de algún tipo de investigación para estudios de posgrado.

Hablamos de gestión, para referirnos a la promoción de determinada actividad -en nuestro caso la investigación- y todas las facetas asociadas. El gestor en el ámbito institucional será el jefe del Departamento de Investigaciones; el profesor a cargo del espacio curricular afín, es el gestor en el nivel áulico, y en el plano individual, el gestor podrá ser el director o tutor de la investigación, o el Jefe de Departamento.

\section{PRINCIPALES OBSTÁCULOS POR ATENDER EN LA GESTIÓN DE LA INVESTIGACIÓN}

En los tres niveles mencionados, una de las principales dificultades se relaciona con la escritura.

Por una parte, tenemos ideas interesantes e incluso, la estructura de la investigación que, sin embargo, permanecen durante mucho tiempo en la "memoria flotante" sin que se plasmen en el papel. El temor a la escritura o el menosprecio por el valor de las propias ideas, es el primer obstáculo. Ante ello, una estrategia fundamental consiste en señalar la importancia del registro escrito, y la certeza de que éste será recibido con respeto y paciencia, por más fragmentaria o provisoria que esta sea. El gestor de la investigación debe valorar el esfuerzo que tales esbozos pudieron haber significado, y mostrar el potencial relacional que estos elementos puedan tener. Cabe recordar las fabulosas posibilidades de la computadora, que permite enriquecer las primeras ideas o palabras sueltas en entramados cada vez mayores, y conservar una copia de cada versión.

Por otra parte, y amén de la propia actividad de escribir, otros sucumben ante la pregunta inicial sobre qué escribir. Al respecto, podríamos resumir la respuesta en dos grupos:

- Uno de ellos consiste en partir de las propias inquietudes o problemas, y desde allí, la situación problemática de la investigación. Un breve lapso de ejercicio en la docencia seguramente proporciona un acervo, pero aun las y los estudiantes, a partir de su experiencia, disponen de un caudal nada despreciable. No obstante, puede que sea 
difícil formular en términos precisos. Al respecto, cabe ejemplificar esta situación con una pwwropuesta que corregí hace poco tiempo, titulada "El lugar del niño hoy". Indudablemente, la persona tenía todo un mundo de ideas que era preciso explicitar tras esa denominación tan general: el lugar, respecto a qué, y si ese hoy se refería a este año, a la última década o al último cuarto de siglo, por ejemplo.

- Otro camino para obtener ideas, es la lectura de bibliografía sobre el tema de interés e incorporar algún aspecto novedoso. Puede que no sea exactamente lo que más interese a título personal, pero las publicaciones reconocidas ayudarán como entrenamiento.

En este punto, pueden presentarse matices que merecen una reflexión: Agrandes rasgos podemos hablar de investigación disciplinar o investigación educativa; investigación de campo, de laboratorio o investigación teórica. Ninguna de ellas tiene una valoración per sé, pero al mismo tiempo, cada una merece consideraciones especiales. Una perspectiva amplia da cabida a cualquiera de ellas en la medida en que aporte algo novedoso a lo que ya se conocía. Naturalmente, no todos comparten los mismos criterios. Si el docente investigador o la institución están exclusivamente interesados en la investigación educativa, es posible convertir una temática disciplinar en educativa, a partir del tratamiento de su enseñanza, y orientarla hacia la didáctica o lo curricular, preguntándose por su inclusión en el currículum de un determinado nivel. Esto requiere del convencimiento del interesado, pues si bien parte de un sustrato común, es otro enfoque.

Otro obstáculo que puede surgir de estas primeras etapas, es la dificultad para la lectura, no sólo por la consabida falta de tiempo o de energías, sino por las carencias bibliográficas o -más importante aún- por la falta de información acerca de bibliografía existente y de sitios dónde buscarla. Me refiero a la poca frecuencia con que se revisen las revistas académicas, por la generalizada razón de que estas requieren de suscripción y los costos son poco accesibles. Sin embargo, también por desconocimiento se desaprovechan las bibliotecas y centros de documentación, como puede comprobarse al consultar al investigador acerca de los sitios visitados o las publicaciones periódicas consultadas.

Aquí cabe un importante señalamiento a los gestores de la investigación -como hemos acordado llamarlos aquí-. Es muy frecuente utilizar libros casi en exclusividad, los cuales, en general, presentan un abordaje consumado de las afirmaciones. Incluso, aquellos que ponen al descubierto la génesis de las conclusiones, como pueden ser los libros de Piaget, en los que se relatan las experiencias con sus hijos, tendemos a focalizar los resultados obtenidos, descuidando la trastienda que da lugar a éstos, y en consecuencia, obturando toda posibilidad de que el lector encuentre esa nueva faceta.

Las revistas, en cambio, se dedican a presentar informes de investigación. Las que lo hacen, dejan ver las secciones habituales de una investigación, ya sea que la investigación se vea como proceso o producto. En aquellos artículos en que estas secciones están encubiertas, es un buen ejercicio reconstruir metodológicamente los pasos de la investigación; es decir, realizar el reconocimiento de objetivos, hipótesis y problema, al interior del escrito.

La lectura de revistas es de utilidad no sólo como andamiaje para el planteamiento de la investigación, sino también para la búsqueda de antecedentes. En la actualidad, existe un instrumento de relativa importancia que es bastante desconocido entre los investigadores (en cursos de grado y de posgrado, tanto en el nivel superior no universitario como el universitario, ha sido el de total desconocimiento). Me refiero al Relevamiento Nacional de Investigaciones Educativas, realizado por la Dirección Nacional de Información y Evaluación de la Calidad Educativa -Área de Investigaciones Educativas-, dependiente del Ministerio de Educación, Ciencia y Tecnología de Argentina. 
Siguiendo someramente el recorrido de una investigación, llegamos al Marco Teórico. En algún sentido, esta sección está exenta de algunos problemas debido a que el tratamiento tradicional de su formación en el nivel superior, consiste en el de transmitir marcos teóricos, autores y libros, Ahora bien, en esta etapa, no estamos libres de dificultades:

- $\quad$ La actualización bibliográfica es una de ellas. A pesar de los sitios de Internet, de librerías y editoriales, el acceso a bibliografía reciente, tanto de manera privada como a través de las bibliotecas, es poco. Al respecto, un dispositivo de utilidad ${ }^{2}$ es la implementación de un Concurso de Recensiones o reseñas bibliográficas. Las recensiones son de gran valor para quien las escribe y para quien las lee. Para el autor, la escritura de esas pocas páginas implica un valioso trabajo de síntesis, luego de una lectura concienzuda de un libro. Para sus lectores, un acercamiento indirecto, que sirve para decidir acerca de la lectura o no de dicho material.

Una vez realizado el trabajo de campo, de gabinete, o de análisis bibliográfico, según el tipo de investigación que se trate, es importante que los datos obtenidos constituyan la materia para nuestras conclusiones. Muchas veces, el fuerte apego a determinadas convicciones hacen que los resultados de la investigación reintroduzcan, elípticamente, nuestros presupuestos, y se tornen inútiles los datos.

También, existen otras cuestiones formales que es preciso vigilar: la mención de las citas al interior del trabajo, la diagramación homogénea de los títulos, la redacción del escrito (puntuaciones, ortografía, etcétera). Esta vigilancia apunta a que la calidad del contenido no quede ofuscada por la falta de claridad. Para el gestor de la investigación, es un desafío conocer el límite de estas exigencias, de manera que su señalamiento no obture o desaliente al investigador. En todo caso, puede reservarse esas indicaciones para un momento en que las principales decisiones ya hayan sido tomadas y esté pendiente la implementación del diseño.

En definitiva, estos aspectos llevan a tomar decisiones respecto de la dinámica de las tutorías, la frecuencia de los encuentros, las lecturas por sugerir y el nivel de exigencia. En la actualidad, y a partir del "boom" de los posgrados, ha aumentado la cantidad de obras referidas a la escritura de investigaciones, tesis, monografías y otros. Sin embargo, el carácter creativo y procesual de esta tarea no nos exime del seguimiento. Aun cuando haya otro profesional que respalde la investigación (como puede ser en el caso de los adscriptos con su profesor a cargo), suele ser interesante la combinación de un experto en metodología de la investigación y un especialista en el tema, con los acuerdos y las jurisdicciones bien clarificadas.

\section{LA DIFUSIÓN DE LAS INVESTIGACIONES}

Cuando hemos atravesado exitosamente este camino, el destino natural y deseable de una investigación es su difusión, y esta se realiza a través de dos canales fundamentales: los eventos académicos y las publicaciones.

Los eventos atentan contra el trabajo solitario del docente, y más aún, de investigaciones realizadas extra muros, en los escasos tiempos libres. Por lo tanto, es de vital importancia la

El mismo fue implementado en el Instituto de Educación Superior No 28 “Olga Cossettini” de Rosario, Argentina en los años 2005 y 2006. 
realización de eventos en los que nos encontremos no hablando sobre los temas que consigna el programa de nuestra materia, sino de aquel que hemos elegido, en tanto esta es una de las bondades de la investigación.

Por otra parte, es recomendable la realización de encuentros internos, tipo ateneos, cuando se quiere lograr un acercamiento al interior de la institución, y a su vez, un diagnóstico del estado de la investigación. Si se trata de un evento asociado con la investigación, es fundamental que los expositores representen su desarrollo, para poner al descubierto la génesis del proceso, y no destinar su intervención sólo a los resultados, a la situación problemática que da origen a la investigación o al desarrollo del marco teórico.

Igualmente, es preciso tener en cuenta que tanto la presentación en un evento como un artículo para revista, requieren que nuestra investigación sea reescrita dentro de límites y estilos. Corresponde a los gestores de la investigación brindar el asesoramiento necesario para que un buen trabajo no se desluzca cuando se pasa de un formato a otro.

Por otra parte, la afinidad temática, o al menos, disciplinar, es un factor fundamental para el aprovechamiento del encuentro. Los eventos miscelánea pueden generar una escucha desatenta del auditorio. A la inversa, el seguimiento de la audiencia y el posterior diálogo, resultan fructíferos.

Las publicaciones constituyen el otro canal de difusión de las investigaciones. Dada esta estrecha asociación, se concibe como una unidad al Departamento de Investigaciones y Publicaciones. Sin embargo, este sintagma resulta muy ambicioso, como veremos al examinar las tareas que las publicaciones conllevan. La combinación responde, inversamente, a la magnitud que el nombre puede acarrear, a cuestiones de escasez presupuestarias que engloban en un único cargo dos tareas relacionadas pero con entidad propia.

Las publicaciones son motivo de diversas interpretaciones:

- $\quad$ Para algunos, la mera exposición de un trabajo es ya digno de tal nombre.

- Otra característica, generalmente asociada con la anterior, es la de reproducción masiva o en grandes cantidades, si pensamos en el soporte papel.

- Para los apegados al formato libro, es la idea de calidad del impreso, dureza de la tapa, gráfica, imagen resulta vulnerable en tiempos de crisis.

Estas características son propias de la publicación. No obstante, hay otras fundamentales, no siempre presentes en el imaginario académico:

- $\quad$ Por una parte, la idea de la evaluación, también aplicada al mundo de las investigaciones: interna, externa o ambas. Interna si se limita a la institución de pertenencia del autor o el investigador, y externa si la trasciende. La presencia de la externa o de ambas jerarquiza el trabajo; por ejemplo, a la hora de realizar el registro de las publicaciones.

- $\quad$ Registro de la propiedad intelectual. En Argentina, la tarea está a cargo del Centro Argentino de Información Científica y Teconológica (Caicyt): Centro Nacional Argentino del ISSN, dependiente del Consejo Nacional de Investigaciones Científicas y Tecnológicas (Conicet). Los libros cuentan con un número de identificación internacional denominado ISBN, siglas para International Standard Book Number, cuya traducción es: Número de libro estandarizado internacional. Las revistas, por su parte, cuentan con un ISSN: International Standard serial number; es decir, Número seriado estandarizado internacional. La idea de serie alude a la aparición periódica de la revista, así como de las series monográficas, a diferencia de la 
aparición única del libro, más allá de las reimpresiones. El trámite para obtenerlo es bastante simple y no tiene costo alguno, y se realiza en el centro mencionado. Sin embargo, requiere la certeza de la pronta aparición de un número de la revista.

La incertidumbre habitual respecto de las posibilidades materiales para producir una revista, hace que muchas circulen sin esta identificación, lo cual, además de la vulnerabilidad en la que quedan, les quita la posibilidad de ser ubicadas en las bases de datos de publicaciones periódicas. Desafortunadamente, un encuentro de editores de revistas académicas argentinas, realizado a fines del 2005 en la ciudad de Rosario, expresaba la poca visibilidad de estas revistas, la escasa circulación y las dificultades económicas.

El financiamiento es otro tema importante para quienes gestionamos las publicaciones institucionales. Mientras que en publicaciones económicamente solventes figura un comité conformado por asesores legales, gerente comercial, diseñadores gráficos y otros, en los Institutos de Formación Docente nos encontramos en cambio un pequeño grupo de profesores realizando todas esas tareas. A su vez, la coyuntura nos expone a situaciones que atentan contra nuestros preconceptos; por ejemplo, el hecho de que los principales auspiciantes sean empresas comerciales y no entidades académicas.

Apartándonos un poco del tema investigaciones, pero fundamental si estamos contemplando la faceta de las publicaciones en Instituciones de Formación Docente, está la concreción de un boletín informativo o figura similar que se edite periódicamente (ya sea en forma virtual, en papel, o en ambos).

El quehacer de las instituciones de Nivel Superior, sean Superior no universitario o universitario, genera una cantidad de propuestas no encuadradas necesariamente en el ámbito de las investigaciones, pero relacionadas con las otras funciones que merecen ser conservadas a modo de crónicas institucionales y difundidas al interior de la comunidad educativa para instituciones de grandes dimensiones, es recomendable la circulación de esta panorámica institucional, pues contribuye al conocimiento recíproco, a la comunicación intrainstitucional y promover iniciativas.

\section{EL COMPLEJO ROL DEL GESTOR DE LAS INVESTIGACIONES Y PUBLICACIONES}

Las tareas mencionadas constituyen un verdadero desafío a los diversos gestores. En el caso del Jefe del Departamento de Investigaciones, la carga horaria con la que cuenta pronto se ve colmada. Ante ello, una propuesta alternativa ${ }^{3}$ consiste en crear la figura del pasante en el Departamento de Investigaciones, con su respectivo reglamento de Pasantía. La figura -inexistente al momento- se monta a partir de la de adscrito, modificando en primer lugar el plazo, y quitándole la fuerte carga de docencia que tiene implícita. El pasante participa de las actividades en curso en el Departamento al momento de su inserción, al tiempo que potencia la proyección de éste.

Otro punto de enriquecimiento de la gestión está dado por el diálogo e intercambio entre colegas, ya mencionado a propósito de los eventos. Es precisamente la intención de este artículo compartir estrategias y suscitar debates entre quienes tenemos la tarea de gestionar el desarrollo de las investigaciones y publicaciones en el Nivel Superior, en contextos de sociedades en vías de desarrollo.

Nuevamente se refiere a una experiencia llevada a cabo en el Instituto Olga Cossettini de Rosario. 


\section{REFERENCIAS}

Centro Argentino de Información Científico y Tecnológica -CAICYT-. (2006). Centro Nacional Argentino de ISSN. Disponible en: http://www.caicyt.gov.ar/issn.

Charreau, E. (2005). Una década en el desarrollo científico tecnológico en América Latina. Ministerio de la Producción, Subsecretaría de Ciencia, Tecnología e Innovación. Provincia de Santa Fe, Argentina.

González, D., Gruschetsky, M., y Serra, J. (2005). La formación docente en el marco de la educación superior no Universitaria. Una aproximación cuantitativa a su oferta de Carreras, capacitación, investigación y extensión. Dirección Nacional de Información y Evaluación de la Calidad Educativa (DINIECE), Área de Investigaciones Educativas, Ministerio de Educación, Ciencia y Tecnología. Argentina.

Pozzo, M. (2006). La investigación en educación artística en Awrgentina: Estado del Arte. Un abordaje desde el Relevamiento Nacional de Investigaciones Educativas. Revista de la Escuela de Ciencias de la Educación. Año 2, No 1.

Serra, J. C., y Landau, M. (2003). Relevamiento Nacional de Investigaciones Educativas 2001-2002. Dirección Nacional de Información y Evaluación de la Calidad Educativa (DINIECE), Argentina: Área de Investigaciones Educativas, Ministerio de Educación, Ciencia y Tecnología. 